\title{
Face Recognition by Classification using Radial Basis Function
}

\author{
Venkata Naresh Mandhala ${ }^{1}$, Debnath Bhattacharyya ${ }^{2}$ and Tai-hoon Kim ${ }^{3}$ \\ ${ }^{1}$ Dept. of Information Technology, VFSTR University \\ Vadlamudi, Guntur, India \\ ${ }^{2}$ Dept. of Computer Science and Engineering, VFSTR University \\ Vadlamudi, Guntur, India \\ ${ }^{3}$ Dept. of Convergence Security, Sungshin Women's University, \\ 249-1, Dongseon-dong 3-ga, Seoul, 136-742, Korea \\ mvnaresh.mca@gmail.com ${ }^{1}$,debnathb@gmail.com ${ }^{2}$,taihoonn@daum.net ${ }^{3}$
}

(Corresponding Author)

\begin{abstract}
The face recognition task involves extraction of unique features from the human face. Manifold learning methods are proposed to project the original data into a lower dimensional feature space by preserving the local neighborhood structure. PCA and LDA are used for the feature extraction and the resultant feature vectors are fused with the different fusion techniques and the proposed method yields good recognition rate with PCA Fusion of PCA and LDA features and those are classified with neural network. In general the size of the face database is too high and it needs more memory and needs more time for training so that to improve time and space complexities there is a need for dimensionality reduction. The extracted features are classified with Neural Network to improve the recognition rate.
\end{abstract}

Keywords: Face recognition, Fusion, LDA, Neural Networks, PCA

\section{Introduction}

Image processing is a form of signal processing for which the input is an image such as video/photo frame. Image processing technique can be used in different formats like image compression, biometric, security considerations from different domains. Most of the image processing [8] techniques testing the image in two dimensional way and then apply standard signal processing techniques to that image. Principal Component Analysis/Linear Discriminant Analysis approaches and back propagation neural networks are used for detecting [9] face recognition, it is the new approach to recognize the faces in less training time and less training patterns. First we use Principal Component Analysis for lower dimensionality of a space for retrieving face space and then to extract these features on high dimensional face space we perform LDA to [1] maximize the discriminatory power for detecting face space regions in image extraction. Principal Component based Analysis technique [9] normally contains two phases: Training and Classification phases, in training phase Eigen-space established from training samples in image extraction process and then training face images are mapped with Eigen space values present in the image extraction.

\section{Background Work}

\subsection{Existing System}

For Face recognition, traditionally more number of methods was developed which are as follows: 
Eigen Faces Method: This schema decomposes images into a small set of characteristic features called 'Eigen Faces' which is based on the principal [5, 6] component of the initial set of face images. Any face can be expressed [3], as linear combinations of the singular vectors of the set of faces, and these singular vectors are eigenvectors of the covariance matrices.

Fisher Faces Method: This schema is based on NWFE method that improves LDA by focusing on sample near the eventual decision boundary location. The main ideas of NWFE put different weights on every sample to [2], compute the "weighted means" and define new nonparametric between-class and within-class scatter matrices. It matrix formation as follows

$$
\begin{gathered}
S^{N W}=\sum_{j=1}^{l} P_{i} \sum_{i=1}^{l} \sum_{k=1}^{N_{i}} \frac{\lambda^{(i, j)}}{N_{i}} *\left(X^{i}-M_{j}\left(X^{j}\right)\left(X^{j}\right)-M_{j}\left(X^{j}\right)\right) T \\
M_{j}\left(x_{i} k\right)=N_{j \_} l=1 w(i, j) k l x_{j} l
\end{gathered}
$$

Where $\mathrm{N}_{\mathrm{i}}$ is the training sample size of class $\mathrm{I}$, $\mathrm{xk}$ is the kth sample class present in the image repository $\mathrm{Mj}\left(\mathrm{x}_{\mathrm{i}} \mathrm{k}\right)$ denotes the weighted mean [7] corresponding to xi $\mathrm{k}$ for class $\mathrm{j}$, and $\operatorname{dist}(\mathrm{x}, \mathrm{y})$ is the distance measured from $\mathrm{x}$ to $\mathrm{y}$. The closer $\mathrm{xi} \mathrm{k}$ and $\operatorname{Mj}(\mathrm{xi} \mathrm{k})$ are, the larger the weight $\lambda(i, j) k$ is. The sum of $\lambda(i, j) k$ for class $i$ is one. In this method we have to implement the LDA development histogram techniques for recognizing face detection.

\subsection{Proposed System}

The proposed system is an appearance based face recognition method called Radial Basis Function approach. This is a new face recognition method based on PCA, LDA and Radial Basis Function is proposed for classification. This method consists of four phases: i) Normalization ii) Feature extraction using PCA and LDA, iii) Feature fusion using PCA and iv) Classification using Neural Networks Radial Basis Function. Combination of PCA and LDA is used for improving the capability of LDA when a few samples of images are available and neural classifier is used to increase classification accuracy.

In the proposed system, 2 different feature domains are extracted from the training set in parallel. Therefore this approach can extract more characteristics of face images for classification purpose. Compared to the conventional classifiers, Neural Networks classifiers gives better results.

2.2.1. Preprocessing or Normalization of a Face Image: The aim of the face preprocessing step is to normalize the coarse face detection, so that a robust feature extraction can be achieved. Depending on the application, face preprocessing includes: Alignment (translation, rotation, scaling) and light normalization / correlation. The face preprocessing step aims at normalizing, i.e. reducing the variation of images obtained during the face detection step.

2.2.2. Feature Extraction: PCA and LDA are two feature extraction techniques used for data reduction and feature extraction in appearance based approaches. In the proposed system we are using both feature extraction techniques in parallel to get the best feature space as shown in Figure 1. The output of the PCA algorithm is called Eigenfaces. The output of LDA algorithm is called Fisher faces. PCA extracts features that represent the class. LDA extracts the features that are required to separate classes. Here in this method both Eigenfaces and fisher faces are extracted to get the advantages of both PCA and LDA. 


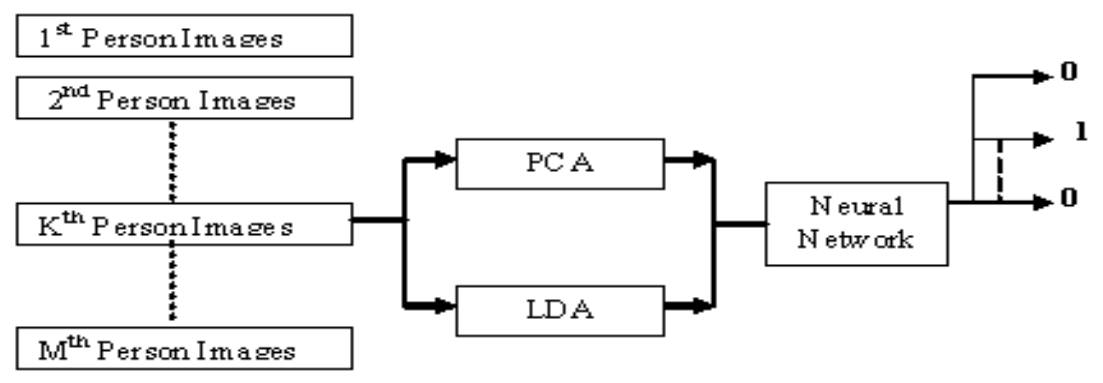

Figure 1. Feature Extraction in Parallel

2.2.3. Feature Fusion: We divide two main processes, which are the training and the testing processes. On the training process, training sets are extracted by PCA, LDA. The results of feature extraction are projected and selected. The selection results of the training process are re-fused into a whole feature. Whereas on the testing process, testing set is projected on each of the projection results of the training sets and selected according to the number of results in the training sets selection.

Fusion of feature extraction results is usually conducted on an appearance global structure only, such as PCA+LDA. We propose an appearance feature selection and fusion of the global structures. An appearance feature extraction with the global structures is resulted by PCA and LDA. Feature extraction results for PCA, LDA respectively can be expressed by using the following equation.

$$
\begin{aligned}
& \wedge P C A=\left[\wedge_{1,1} ; \wedge_{1,2} ; \ldots ; \wedge_{1, m}\right] \\
& \wedge L D A=\left[\wedge_{2,1} ; \wedge_{2,2} ; \ldots ; \wedge_{2, m}\right]
\end{aligned}
$$

If number of features extracted for each the feature extraction method is $\mathrm{m}$, whereas the number of feature selection is $S$ and $S<m$, than number of features fusion of PCA+LDA can be represented by $2 \mathrm{~S}$. The result of feature fusion can be written in the following matrix.

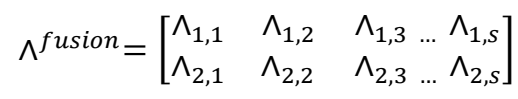

To simplify calculation, the above Equation can be written in the row vector as shown in the following equation:

$$
\Lambda^{\text {fusion }}=\left[\wedge_{1,1} ; \wedge_{1,2} ; \wedge_{1,3} \ldots \wedge_{1, s} ; \wedge_{1, s * 1} ; \wedge_{1, s * 2} ; \ldots \Lambda_{1,2 s}\right]
$$

2.2.4. Classification using Radial Basis Function: The idea behind Radial Basis Function (RBF) Networks, shortly RBF, is based on Cover's theorem: "A complex pattern-classification problem cast in a high-dimensional space nonlinearly is more likely to be linearly separable than in a low-dimensional space".

\subsection{Training}

As MLPs, RBF Networks are trained using a labeled training data set and by adjusting its parameters such that an error function is minimized. In most cases, the error function is the sum over all training examples of the squared distances between the NN output and the desired output. However, different training strategies can be adopted with respect to the hidden layer: 
1. Use fixed Gaussian RBFs chosen at random.

2. Use RBFs with fixed variance and adjust their centers by an unsupervised learning algorithm.

3. Adjust centers and variances of the RBFs by a supervised learning algorithm.

\section{Testing and Comparison Study}

There are virtually no tools to help us select an appropriate architecture and learning parameters for a neural network. In most cases, learning parameters are determined by experience or based on the trial and error method. We may work both ways to select our best set of parameters:

\subsection{Training and Testing of Neural Networks}

PCA features and LDA features are added together to send to the neural network. Only one neural network is used for classification. ORL face database is used for training and testing. The training is performed by $\mathrm{n}$ poses from each subject and the performance testing is performed by $10-\mathrm{n}$ poses of the same subjects.

After calculating the Eigenfaces using PCA the projection vectors are calculated for the training set and the results are called Eigen features. Similarly, after calculation of the fisher faces using the LDA, projection vectors are calculated for the training set and are called fisher faces.

Then these two results are combined using the + operator and then send to the PCA fusion and later for classification using neural network. When a new image from the test set is considered for recognition, the image is mapped to the Eigen space and fisher space and then the results are added. Hence, the image is assigned to a feature vector. Each feature vector is fed to its respective neural network and the network outputs are compared.

\subsection{System Performance}

The performances of the proposed systems are measured by varying the number of faces of each subject in the training and test faces. Table 1 shows the performances of the proposed PCA-NN and LDA-NN methods based on the neural network classifiers as well as the performances of the conventional PCA and LDA based on the Euclidean Distance classifier. Along with the existing algorithms the Table 1 also shows the performance of the proposed method.

The result clearly shows that the proposed recognition system 2 FNN, outperforms, the existing PCA-NN and LDA-NN, and also outperforms the conventional PCA and LDA based recognition systems. The $2 \mathrm{FNN}$ shows the highest recognition performance, where this performance is obtained because of the fact that the $2 \mathrm{FNN}$ method discriminate the classes better and also describes the classes better than the existing ones. Above results are shown in the Graph 1.

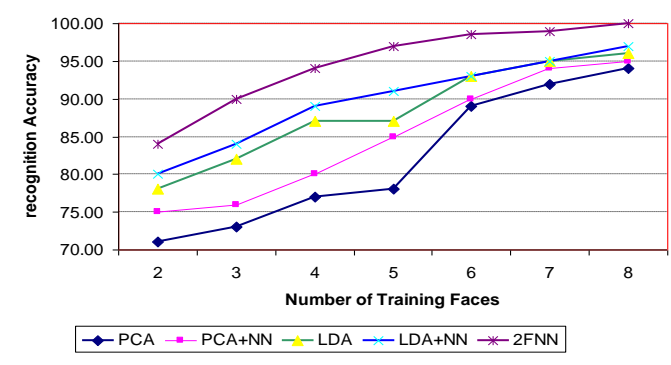

\section{Graph 1. Accuracy Increases with the Increase in Number of Training Images}




\subsection{Comparison with previous FR methods}

The sample face images are shown in Figure 6, The face images which are used for training and testing the neural network represent persons of various ethnicities, age and gender. A total of 400 face images of 40 persons with different facial expressions are used from the ORL face database (AT\&T Laboratories Cambridge, online resources).

Compared to the existing methods the proposed RBF face recognition achieved $98.5 \%$ accuracy. The Table 2 shows the results.

Graphical representation of the above test results are shown in the Graph 2. Here we can see improvement of the proposed system over the existing systems.

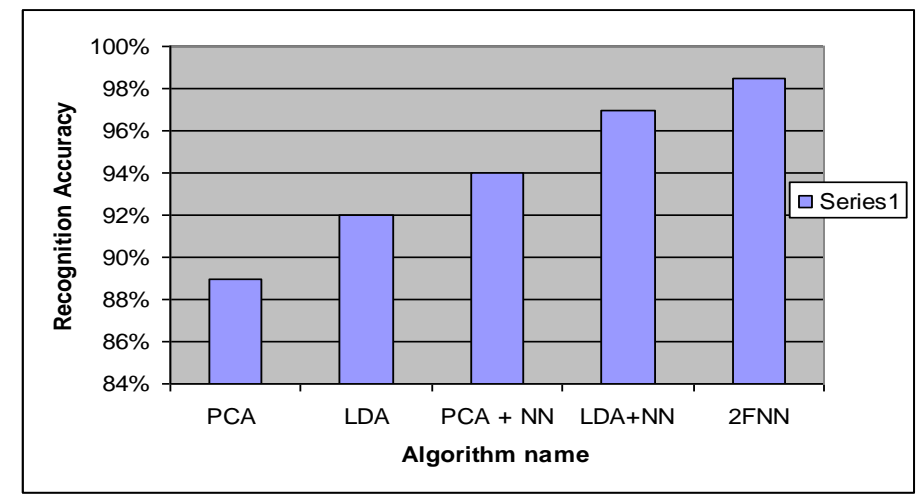

\section{Graph. 2. Accuracy of the Proposed and Existing Algorithms}

\subsection{Total Error Rate}

The purpose of the experiment is to evaluate the performance of the face recognition system by applying Neural Networks. The face images are frontal face images, which are taken from our ORL face images database. The database consists of face images from forty individuals, each with ten face images.

The proposed $2 \mathrm{FNN}$ scores very low error rates than the previous methods shown in Table 3. False acceptance rate of the proposed system is 1.050 and false rejection rate is 3.430. So the total error rate of the proposed system is 2.240 .

Graph 3 shows the total error rate in existing methods. We can see the error rate is very low in the proposed method as compared to the existing methods.

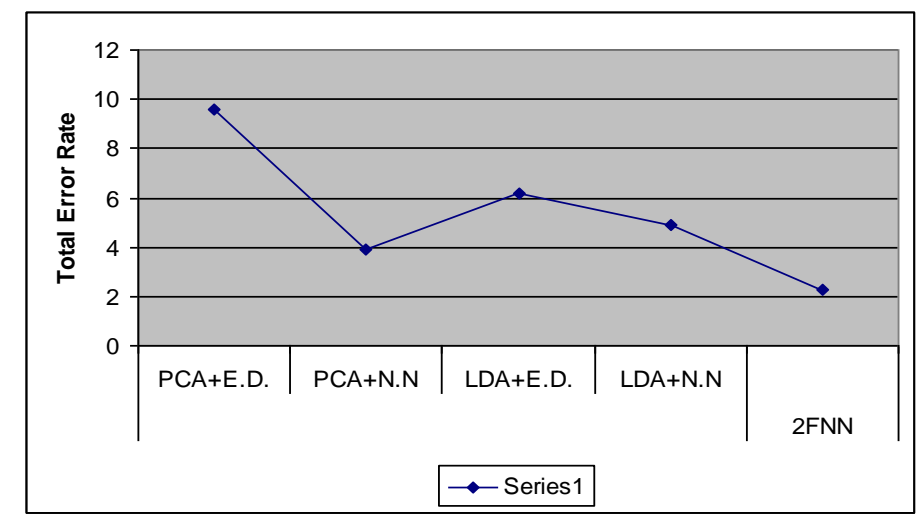

\section{Graph. 3. Values of FAR, FRR and TER with Various Classifiers}

Here Graph 4 shows the accuracy of the proposed method. By seeing the following graph we can easily understand that the accuracy of the proposed system is improved when the number of images in the training set increases. 


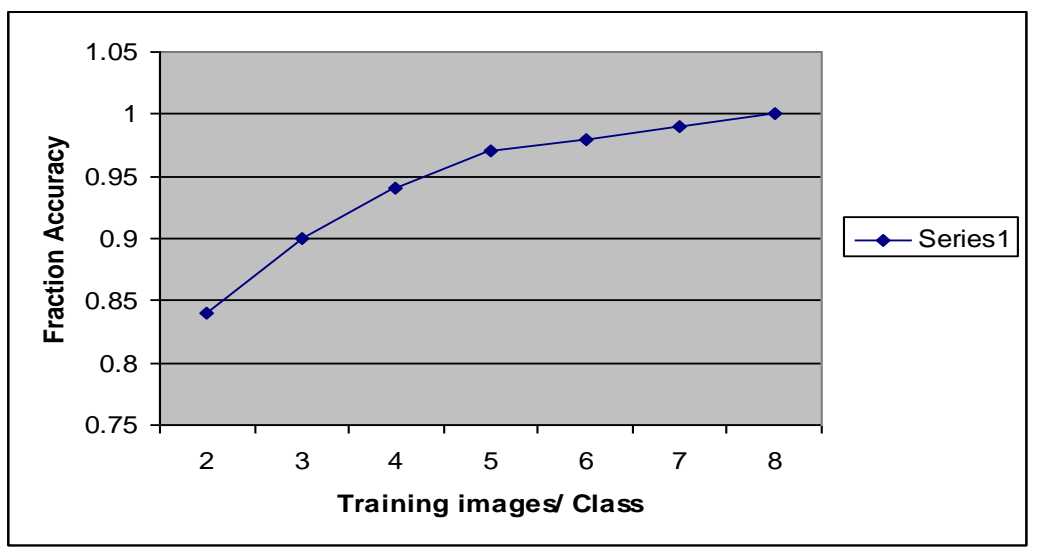

Graph 4. Accuracy of the Proposed Method

\subsection{Performance Across Databases}

Proposed system is tested across various bench mark databases. Test result of recognition is shown in the following Table 4 and Graph 6.

Our proposed system gains $98.5 \%$ accuracy on ORL database. It scored $97.76 \%$ accuracy on Indian face database and scored $97 \%$ of accuracy on Yale face database.

The above results are shown in a Graph 5 for better representation and understanding.

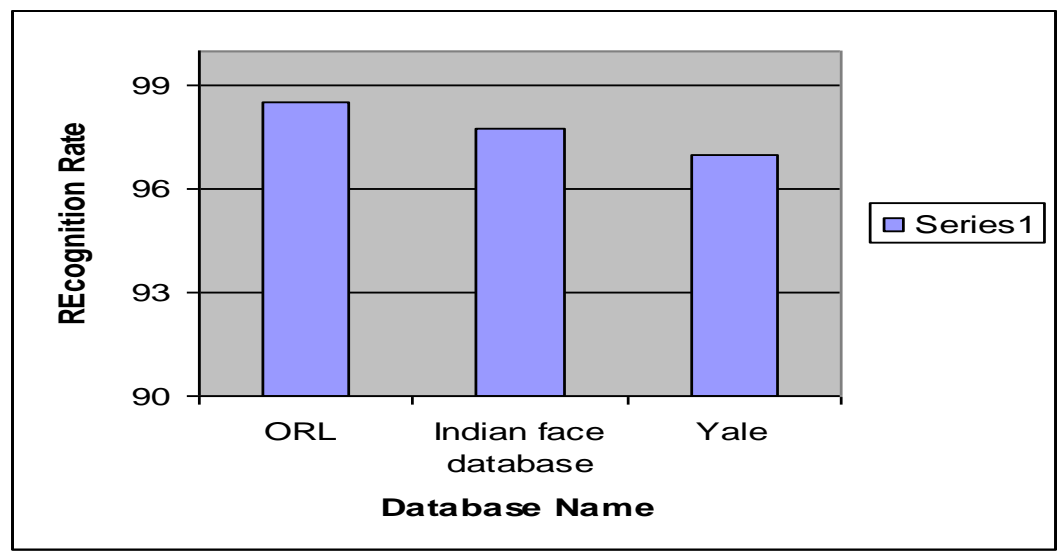

\section{Graph 5. Recognition across Databases}

\section{Conclusions}

In this paper, different face recognition techniques have been implemented using hybrid approach and multi-scaling of facial components concept for feature extraction, PCA and LDA are used for the feature extraction and the resultant feature vectors are fused with the different fusion techniques and the proposed method yields good recognition rate with PCA Fusion of PCA and LDA features and those are classified with the RBF neural network. The proposed method has tested with ORL database and it gives $98.5 \%$ of the recognition rate.

Table 1. Proposed System Recognition Comparison with Existing Systems

\begin{tabular}{|c|l|l|l|c|c|c|}
\hline $\begin{array}{l}\text { Traini } \\
\text { Img } \\
\text { Images }\end{array}$ & $\begin{array}{l}\text { Testing } \\
\text { Images }\end{array}$ & PCA & PCA-NN & LDA & LDA-NN & RBF 2FNN \\
\hline 2 & 8 & 71 & 75 & 78 & 80 & 84 \\
\hline
\end{tabular}




\begin{tabular}{|c|c|c|c|c|c|c|}
\hline 3 & 7 & 73 & 76 & 82 & 84 & 90 \\
\hline 4 & 6 & 77 & 80 & 87 & 89 & 94 \\
\hline 5 & 5 & 78 & 85 & 87 & 91 & 97 \\
\hline 6 & 4 & 89 & 90 & 93 & 93 & 98.5 \\
\hline 7 & 3 & 92 & 94 & 95 & 95 & 99 \\
\hline 8 & 2 & 94 & 95 & 96 & 97 & 100 \\
\hline
\end{tabular}

Table 2. Average Rate of Success in Proposed and Existing Systems

\begin{tabular}{|c|c|}
\hline Algorithm & $\begin{array}{c}\text { Average Rate of Success Face } \\
\text { Recognition }\end{array}$ \\
\hline PCA+E.D. & $89 \%$ \\
\hline LDA+E.D. & $92 \%$ \\
\hline PCA + NN & $94 \%$ \\
\hline LDA+NN & $97 \%$ \\
\hline $\begin{array}{c}\text { PCA+LDA+RBF } \\
(2 \mathrm{FNN})\end{array}$ & $98.50 \%$ \\
\hline
\end{tabular}

Table 3. Values of FAR, FRR and TER with Various Classifiers

\begin{tabular}{|c|c|c|c|c|}
\hline Feature Extractor & Classifier & FAR & FRR & TER(\%) \\
\hline \multirow{2}{*}{ PCA } & E.D & 6.540 & 12.590 & 9.565 \\
\cline { 2 - 5 } & N.N & 4.140 & 3.700 & 3.920 \\
\hline \multirow{2}{*}{ LDA } & E.D & 6.030 & 6.300 & 6.165 \\
\cline { 2 - 5 } & N.N & 4.660 & 5.190 & 4.925 \\
\hline \multicolumn{2}{|c|}{ 2FNN } & 1.050 & 3.430 & 2.240 \\
\hline
\end{tabular}

Table 4. Comparison of Recognition in Three Databases

\begin{tabular}{|c|c|c|c|}
\hline Database & Number of images & Correct recognition & Recognition rate \\
\hline ORL & 400 & 394 & 98.5 \\
\hline Indian face database & 600 & 586 & 97.76 \\
\hline Yale & 800 & 776 & 97 \\
\hline
\end{tabular}

\section{References}

[1] M. Agrawal, H N. Jain and M. Kumar, "Face Recognition using Principle Component Analysis", Eigenface and Neural Network, International Conference on Signal Acquisition and Processing, Bangalore, February 9-10 (2010), pp. 310-314.

[2] L. Diago, T. Kitaoka, I. Hagiwara and T. Kambayashi, "Neuro-Fuzzy Quantification of Personal Perceptions of Facial Images Based on a Limited Data Set", IEEE Transactions on Neural Networks, (2011) December, vol. 22, no. 12, pp. 2422-2434.

[3] H. Kobayashi and Q. Zhao, "Face Detection with Clustering, LDA and NN", IEEE International Conference on Systems, Man and Cybernetics, Montreal, Quebec, October 7-10 (2007), pp. 1670-1675.

[4] C. Boehnen, D. Barstow, D. Patlolla and C. Mann, "A multi-sample standoff multimodal biometric system”, IEEE Fifth International Conference on Biometrics: Theory, Applications and Systems (BTAS), (2012) September 23-27, pp. 127-134. 
[5] H.-M. Moon and S. B. Pan, "The LDA-Based Face Recognition at a Distance Using Multiple Distance Image", Seventh International Conference on Innovative Mobile and Internet Services in Ubiquitous Computing (IMIS), (2013) July 3-5, pp. 249-255.

[6] V. P. Kshirsagar, M. R. Baviskar and M. E. Gaikwad, "Face recognition using Eigenfaces", 3rd International Conference on Computer Research and Development (ICCRD), vol. 2, no. 3, (2011) March 11-13, pp. 302-306.

[7] Sahoolizadeh, A. Hossein, B. Zargham Heidari, and C. Hamid Dehghani, "A New Face Recognition Method using PCA, LDA and Neural Network", International Journal of Computer Science \& Engineering (2008), vol. 2, no. 4, p. 7.

[8] M. Sharif, S. Mohsin, M. J. Jamal and M. Raza, "Illumination Normalization Preprocessing for Face Recognition", International Conference on Environmental Science and Information Application Technology (ESIAT), Wuhan, vol. 2, (2010) July 17-18, pp. 44-47.

[9] S. Venkatrama Phani Kumar, K. V. K. Kishore and K. H. Kumar, "Hybrid Face Recognition with Locally Discriminating Projection", International Conference on Signal Acquisition and Processing, Bangalore, (2010) February 9-10, pp. 327-331. 